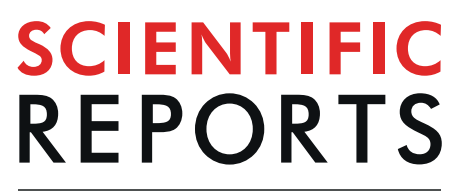

natureresearch

\title{
OPEN Growth patterns during early childhood in children born small for gestational age and moderate \\ preterm
}

Received: 28 January 2019

Accepted: 23 July 2019

Published online: 09 August 2019

\author{
Linda Lindström ${ }^{1}{ }^{1}$, Fredrik Ahlsson ${ }^{1}$, Maria Lundgren ${ }^{1}$, Eva Bergman ${ }^{1}$, Erik Lampa ${ }^{2}$ \& \\ Anna-Karin Wikström ${ }^{1}$
}

Today we lack knowledge if size at birth and gestational age interact regarding postnatal growth pattern in children born at 32 gestational weeks or later. This population-based cohort study comprised 41,669 children born in gestational weeks 32-40 in Uppsala County, Sweden, between 2000 and 2015. We applied a generalized least squares model including anthropometric measurements at 1.5, 3, 4 and 5 years. We calculated estimated mean height, weight and BMI for children born in week $32+0$, $35+0$ or $40+0$ with birthweight $50^{\text {th }}$ percentile (standardized appropriate for gestational age, sAGA) or $3^{\text {rd }}$ percentile (standardized small for gestational age, sSGA). Compared with children born sAGA at gestational week $40+0$, those born sAGA week $32+0$ or $35+0$ had comparable estimated mean height, weight and BMI after 3 years of age. Making the same comparison, those born sSGA week $32+0$ or $35+0$ were shorter and lighter with lower estimated mean BMI throughout the whole follow-up period. Our findings suggest that being born SGA and moderate preterm is associated with short stature and low BMI during the first five years of life. The association seemed stronger the shorter gestational age at birth.

Being born small for gestational age (SGA) is associated with increased risk of metabolic and cardiovascular complications as well as suboptimal cognitive development later in life ${ }^{1,2} .85-90 \%$ of children born SGA show linear catch-up growth during early childhood and will to a large extent reach normal adult stature ${ }^{3}$. However, catch-up growth may have both negative and positive effects on future health. The risk of obesity and cardiovascular disease increases, whereas cognitive impairment decreases after rapid catch-up growth ${ }^{4-7}$.

Children born preterm are more often born SGA than those born term ${ }^{8,9}$. Birth at gestational week $32-36$, referred to as moderate to late preterm birth, accounts for approximately $85 \%$ of all preterm births. Despite this, most research on preterm birth involves children born before gestational week $32^{10}$.

Slow neonatal weight gain is common in preterm born children of all birthweights, and is more pronounced the lower the gestational age ${ }^{11,12}$. Hence, not only children born SGA, but also children born appropriate for gestational age (AGA) but preterm may experience a period of perinatal growth restriction. Rapid catch-up growth seems to promote cognitive development in children born moderate preterm, but the evidence regarding metabolic outcomes is scarce in this group ${ }^{13,14}$.

Deviating postnatal growth is more common in children born SGA with comorbidity and short gestational age $^{15,16}$. Earlier research on postnatal growth in children born preterm often comprises study populations of diverse gestational age and birthweight. With substantial neonatal growth restriction and need of intensive care in the most preterm born infants, potential adverse effects might vary widely within study populations including extreme as well as late preterm births ${ }^{17}$.

In this population-based study we had the opportunity to study postnatal growth patterns until five years of age in almost 42,000 children born between gestational week 32 and 40 . We hypothesized that SGA and gestational age at birth may interact on postnatal growth patterns in children born moderate preterm. Further, that body proportions at five years may differ depending on size and gestational age at birth.

${ }^{1}$ Department of Women's and Children's Health, Uppsala University, Uppsala, Sweden. ${ }^{2}$ Uppsala Clinical Research Center, UCR, Uppsala University, Uppsala, Sweden. Correspondence and requests for materials should be addressed to L.L. (email: linda.lindstrom@kbh.uu.se) 


\begin{tabular}{|c|c|c|c|c|c|c|}
\hline & \multicolumn{5}{|l|}{ Gestational Age } & \multirow[b]{2}{*}{ P-value } \\
\hline & Total $(n=41,669)$ & $\begin{array}{l}\text { 32-34 weeks } \\
(\mathrm{n}=532)\end{array}$ & $\begin{array}{l}\text { 35-36 weeks } \\
(\mathrm{n}=1692)\end{array}$ & $\begin{array}{l}\text { 37-38 weeks } \\
(\mathrm{n}=11,271)\end{array}$ & $\begin{array}{l}\text { 39-40 weeks } \\
(\mathrm{n}=28,174)\end{array}$ & \\
\hline \multicolumn{7}{|l|}{ Maternal Characteristics } \\
\hline Age, years, mean $(\mathrm{SD})$ & $30.4(5.1)$ & $30.0(5.4)$ & $30.0(5.3)$ & $30.7(5.2)$ & $30.3(5.0)$ & $<0.05$ \\
\hline Height, cm, mean (SD) & $166.4(6.4)$ & $165.6(5.9)$ & $165.8(6.5)$ & $165.8(6.4)$ & $166.7(6.4)$ & $<0.05$ \\
\hline missing, $\mathrm{n}(\%)$ & $1324(3.2 \%)$ & $24(4.5 \%)$ & $65(3.8 \%)$ & $361(3.2 \%)$ & $874(3.1 \%)$ & \\
\hline BMI early pregnancy, $\mathrm{kg} / \mathrm{m}^{2}$, mean (SD) & $24.6(4.5)$ & $25.1(4.8)$ & $24.7(4.7)$ & $24.8(4.8)$ & $24.5(4.4)$ & $<0.05$ \\
\hline missing, $\mathrm{n}(\%)$ & $4003(9.6 \%)$ & $56(10.5 \%)$ & $174(10.3 \%)$ & $1111(9.9 \%)$ & $2662(9.4 \%)$ & \\
\hline Parity, mean (SD) & $1.9(1.0)$ & $1.7(1.0)$ & $1.8(1.1)$ & $2.0(1.1)$ & $1.9(1.0)$ & $<0.05$ \\
\hline \multicolumn{7}{|l|}{ Country of birth ${ }^{\mathrm{a}}, \mathrm{n}(\%)$} \\
\hline Nordic country, n (\%) & $34,415(82.6 \%)$ & $450(84.6 \%)$ & $1418(83.8 \%)$ & $9196(81.6 \%)$ & $23,351(82.9 \%)$ & \\
\hline Europe, North America, n (\%) & $1489(3.6 \%)$ & $25(4.7 \%)$ & $45(2.7 \%)$ & $377(3.3 \%)$ & $1042(3.7 \%)$ & \\
\hline other, $\mathrm{n}(\%)$ & $5741(13.8 \%)$ & $57(10.7 \%)$ & $228(13.5 \%)$ & $1688(15.0 \%)$ & $3768(13.4 \%)$ & \\
\hline missing, $\mathrm{n}(\%)$ & $24(0.1 \%)$ & 0 & $1(0.1 \%)$ & $10(0.1 \%)$ & $13<0.1 \%)$ & \\
\hline Level of education, years & & & & & & $<0.05$ \\
\hline$\leq 9, \mathrm{n}(\%)$ & $3753(9.0 \%)$ & $48(9.0 \%)$ & $179(10.6 \%)$ & $1107(9.8 \%)$ & $2419(8.6 \%)$ & \\
\hline $10-14, \mathrm{n}(\%)$ & $20,528(49.3 \%)$ & $268(50.4 \%)$ & $864(51.1 \%)$ & $5702(50.6 \%)$ & $13,694(48.6 \%)$ & \\
\hline$\geq 15, \mathrm{n}(\%)$ & $16,698(40.1 \%)$ & $209(39.3 \%)$ & $623(36.8 \%)$ & $4267(37.9 \%)$ & $11,599(41.2 \%)$ & \\
\hline missing, $\mathrm{n}(\%)$ & $690(1.7 \%)$ & $7(0.5 \%)$ & $26(1.5 \%)$ & $195(1.7 \%)$ & $462(1.6 \%)$ & \\
\hline Cohabitation in early pregnancy, $\mathrm{n}(\%)$ & $37,292(89.5 \%)$ & $462(86.8 \%)$ & $1507(89.1 \%)$ & $10,019(88.9 \%)$ & $25,304(89.8 \%)$ & $<0.05$ \\
\hline missing, $\mathrm{n}(\%)$ & $2698(6.5 \%)$ & $38(7.1 \%)$ & $102(6.0 \%)$ & $772(6.8 \%)$ & $1786(6.3 \%)$ & \\
\hline Diabetic disease during pregnancy, n (\%) & $785(1.9 \%)$ & $26(4.9 \%)$ & $87(5.1 \%)$ & $373(3.3 \%)$ & $299(1.1 \%)$ & $<0.05$ \\
\hline Smoking pregnancy week 32, n (\%) & $2752(6.6 \%)$ & $35(6.6 \%)$ & $151(8.9 \%)$ & $848(7.5 \%)$ & $1718(6.1 \%)$ & $<0.05$ \\
\hline missing, $\mathrm{n}(\%)$ & $1395(3.3 \%)$ & $22(4.1 \%)$ & $75(4.4 \%)$ & $372(3.3 \%)$ & $926(3.3 \%)$ & \\
\hline \multicolumn{7}{|l|}{ Infant Characteristics } \\
\hline Birthweight in standard deviation score ${ }^{\mathrm{b}}$, mean (SD) & $0.28(0.99)$ & $0.22(0.98)$ & $0.28(0.97)$ & $0.31(1.01)$ & $0.27(0.98)$ & $<0.05$ \\
\hline Birthweight $<10^{\text {th }}$ percentile ${ }^{\mathrm{b}}, \mathrm{n}(\%)$ & $2109(5.1 \%)$ & $37(7.0 \%)$ & $83(4.9 \%)$ & $589(5.2 \%)$ & $1400(5.0 \%)$ & \\
\hline Birthweight $<3^{\text {rd }}$ percentile ${ }^{\mathrm{b}}, \mathrm{n}(\%)$ & $635(1.5 \%)$ & $13(2.4 \%)$ & $36(2.1 \%)$ & $185(1.6 \%)$ & $401(1.4 \%)$ & \\
\hline Birth length in standard deviation score ${ }^{\mathrm{b}}$, mean (SD) & $0.36(1.01)$ & $0.00(0.98)$ & $0.12(0.93)$ & $0.29(0.99)$ & $0.41(1.01)$ & $<0.05$ \\
\hline Male gender, $\mathrm{n}(\%)$ & $20,785(49.9 \%)$ & $267(50.2 \%)$ & $858(50.7 \%)$ & $5621(49.9 \%)$ & $14,039(49.8 \%)$ & 0.92 \\
\hline Apgar score at $5 \mathrm{~min}$ & & & & & & $<0.05$ \\
\hline $0-6, \mathrm{n}(\%)$ & $333(0.8 \%)$ & $34(6.4 \%)$ & $42(2.5 \%)$ & $77(0.7 \%)$ & $180(0.6 \%)$ & \\
\hline $7-10, \mathrm{n}(\%)$ & $41,055(98.5 \%)$ & $493(92.7 \%)$ & $1633(96.5 \%)$ & $11,110(98.6 \%)$ & $27,819(98.7 \%)$ & \\
\hline missing, $\mathrm{n}(\%)$ & $281(0.7 \%)$ & $5(0.94 \%)$ & $17(1.0 \%)$ & $84(0.7 \%)$ & $175(0.6 \%)$ & \\
\hline No breastfeeding at age two months, $\mathrm{n}(\%)$ & $1298(3.1 \%)$ & $26(4.9 \%)$ & $77(4.6 \%)$ & $392(3.5 \%)$ & $803(2.9 \%)$ & $<0.05$ \\
\hline missing, $\mathrm{n}(\%)$ & $15,558(37.3 \%)$ & $208(39.1 \%)$ & $644(38.1 \%)$ & $4291(38.1 \%)$ & $10,415(37.0 \%)$ & \\
\hline
\end{tabular}

Table 1. Characteristics of study population. ${ }^{a}$ Asia, Africa, South America, former Soviet Union, Oceania. ${ }^{b}$ Birthweight and birth length for gestational age and sex according to the Swedish reference for birthweight, mean in standard deviation scores ${ }^{35}$.

\section{Results}

Of the 41,669 children included in the study, 532 (1.3\%) were born at 32-34 gestational weeks, 1692 (4.1\%) at 35-36 weeks, $11,271(27.0 \%)$ at $37-38$ weeks and 28,174 (67.6\%) at 39-40 weeks. Birthweight and birth length for gestational age and sex were the lowest in children born moderate preterm (32-34 weeks), with p-value for overall difference between groups $<0.05$. Children born in late term pregnancy (39-40 weeks) had the tallest mothers, $1 \mathrm{~cm}$ taller mean height than the other groups, $\mathrm{p}<0.05$. The rate of maternal smoking was the highest in children born late preterm (35-36 weeks). Birthweight and birth length for gestational age and sex were the lowest in children born moderate preterm (32-34 weeks), $\mathrm{p}<0.05$. In all gestational ages, a large proportion of the children had missing information on breastfeeding at age two months. The characteristics of the study population are summarized in Table 1.

The percentage of missing values across the independent variables varied between 0 and $37 \%$. In total, 18,694 (45\%) children had incomplete records. Breastfeeding at two months had 15,558 (37\%) incomplete records, followed by maternal BMI with 4403 (10\%) incomplete records. Maternal BMI and breastfeeding at two months were simultaneously missing in 1482 (4\%) children and maternal smoking and breastfeeding were missing in 630 (2\%) children.

Table 2 and Fig. 1 illustrate differences in estimated mean height and weight at ages 1.5 and 5 years between children born $s$ AGA (birthweight $50^{\text {th }}$ percentile) or $s$ SGA (birthweight $3^{\text {rd }}$ percentile) at gestational week $32+0$ and $35+0$ and children born $s$ AGA at week $40+0$ (reference). At 1.5 years, children born $s$ AGA at week $32+0$ or $35+0$ were shorter compared with children born $s$ AGA at $40+0$ weeks, but estimated heights were comparable between the groups from 3 years of age. At 1.5 years, children born $s S G A$ at week $32+0$ and $35+0$ were both shorter and lighter than the reference group, with a pattern of greater differences the more preterm born. Children born $s$ SGA at $32+0$ were $3 \mathrm{~cm}$ shorter and $1.5 \mathrm{~kg}$ lighter at 1.5 years, corresponding to $-1.1 \mathrm{SD}$ and 


\begin{tabular}{|c|c|c|c|c|}
\hline & \multicolumn{2}{|l|}{ Height difference ${ }^{a}$} & \multicolumn{2}{|l|}{ Weight difference ${ }^{a}$} \\
\hline & In cm $(95 \% \mathrm{CI})$ & In SD (95\% CI) & In $\mathrm{kg}(95 \% \mathrm{CI})$ & In SD (95\% CI) \\
\hline \multicolumn{5}{|c|}{1.5 years } \\
\hline \multicolumn{5}{|c|}{ Born $s$ AGA } \\
\hline $32+0$ & $-1.02(-1.47 ;-0.57)$ & $-0.36-(0.53 ;-0.20)$ & $-0.35(-0.61 ;-0.09)$ & $-0.29(-0.51 ;-0.08)$ \\
\hline $35+0$ & $-0.64(-0.87 ;-0.40)$ & $-0.23(-0.31 ;-0.14)$ & $-0.21(-0.34 ;-0.08)$ & $-0.18(-0.28 ;-0.07)$ \\
\hline \multicolumn{5}{|c|}{ Born $s$ SGA } \\
\hline $32+0$ & $-3.20(-4.43 ;-1.97)$ & $-1.14(-1.58 ;-0.70)$ & $-1.66(-2.37 ;-0.95)$ & $-1.38(-1.98 ;-0.79)$ \\
\hline $35+0$ & $-2.06(-2.68 ;-1.45)$ & $-0.74(-0.96 ;-0.52)$ & $-1.26(-1.61 ;-0.91)$ & $-1.05(-1.34 ;-0.76)$ \\
\hline $40+0$ & $-0.33(-0.60 ;-0.06)$ & $-0.12(-0.21 ;-0.02)$ & $-1.05(-1.42 ;-0.68)$ & $-0.50(-0.63 ;-0.37)$ \\
\hline \multicolumn{5}{|c|}{5 years } \\
\hline \multicolumn{5}{|c|}{ Born sAGA } \\
\hline $32+0$ & $0.33(-0.17 ; 0.84)$ & $0.08(-0.04 ; 0.19)$ & $-0.15(-0.45 ; 0.14)$ & $-0.07(-0.2 ; 0.06)$ \\
\hline $35+0$ & $0.25(-0.01 ; 0.51)$ & $0.06(-0.00 ; 0.12)$ & $-0.08(-0.23 ; 0.07)$ & $-0.03(-0.1 ; 0.03)$ \\
\hline \multicolumn{5}{|c|}{ Born sSGA } \\
\hline $32+0$ & $-1.84(-3.16 ;-0.53)$ & $-0.42(-0.72 ;-0.12)$ & $-2.33(-3.09 ;-1.58)$ & $-1.01(-1.34 ;-0.69)$ \\
\hline $35+0$ & $-1.02(-1.68 ;-0.37)$ & $-0.23(-0.38 ;-0.08)$ & $-1.69(-2.06 ;-1.31)$ & $-0.73(-0.9 ;-0.57)$ \\
\hline $40+0$ & $-0.37(-0.67 ;-0.07)$ & $-0.08(-0.15 ;-0.02)$ & $-1.61(-2.01 ;-1.20)$ & $-0.34(-0.41 ;-0.26)$ \\
\hline
\end{tabular}

Table 2. Differences in estimated mean height and weight. Children born standardized appropriate for gestational age ( $s$ AGA) or small for gestational age ( $s$ SGA) in gestational weeks $32+0,35+0$ and $40+0$ compared with children born $s$ AGA in gestational week $40+0 . s$ AGA defined as birthweight $50^{\text {th }}$ percentile and $s S G A$ as birthweight $3^{\text {rd }}$ percentile of expected for gestational age and sex according to the Swedish reference standards for birthweight, respectively ${ }^{35}$. ${ }^{a}$ Model adjusted for maternal height, BMI, country of birth, maternal diabetic disease during pregnancy, smoking in pregnancy week 32 and breastfeeding at age two months.

$-1.4 \mathrm{SD}$ in height and weight, compared with children born $s$ AGA at $40+0$ (reference). The differences in estimated height and weight between $s$ SGA at $32+0$ or $35+0$ and the reference group remained significant over the follow-up period of 5 years.

Table 3 and Fig. 2 illustrate differences in the estimated height and weight at the same follow-up ages, but children born $s$ AGA (birthweight $50^{\text {th }}$ percentile) and $s$ SGA (birthweight $3^{\text {rd }}$ percentile) at the same gestational age are compared. In comparison with children born sAGA at week $32+0,35+0$ and $40+0$, those born sSGA at the corresponding gestational age had shorter estimated mean heights at all follow-up ages. There was a trend of more pronounced differences in children born in week $32+0$ than $35+0$, which lasted over the follow-up period. A pattern of larger differences the shorter the gestational age was seen.

Table 4, Figs 1 and 2 illustrate the ratio of estimated mean BMI at ages 1.5 and 5 years. Children born $s$ AGA (birthweight $50^{\text {th }}$ percentile) of all gestational ages had comparable BMIs throughout the follow-up period. Children born $s$ SGA (birthweight $3^{\text {rd }}$ percentile) of all gestational ages had lower estimated mean BMI at all follow-up ages compared with children born $s$ AGA at $40+0$. At 5 years, children born $s S G A$ at $32+0,35+0$ and $40+0$ had $9 \%, 7 \%$ and $4 \%$ lower estimated mean BMI than those born sAGA at $40+0$, respectively. Also if we compared children born $s$ AGA and $s$ SGA of the same gestational age, children born sSGA had lower estimated mean BMI at age 5 years than those born $s$ AGA.

At 5 years of age, the proportion of children with a normal BMI $\left(10^{\text {th }}-90^{\text {th }}\right.$ percentile $)$ varied according to SGA-status and gestational age at birth, see Fig. 3. Children born SGA $\left(<10^{\text {th }}\right.$ percentile $)$ and moderate preterm (32-34 weeks) less often had a normal BMI compared with children born AGA ( $10^{\text {th }}-90^{\text {th }}$ percentile) and late term $(39-40$ weeks) $(\mathrm{p}<0.05)$. There was a J-shaped pattern among children born SGA and moderate preterm with a tendency of more subjects with high BMI, but even more children with low BMI at 5 years.

\section{Conclusions}

Statement of principal findings. In children born moderate to late preterm we could show a difference in postnatal growth depending on coexistence of SGA birth or not. Children born AGA and moderate to late preterm had comparable estimated mean height, weight and BMI as children born AGA and term after 3 years of age. Children born SGA and moderate to late preterm were shorter and lighter and had a lower BMI than children born AGA and term throughout the whole follow-up period of 5 years. There was a pattern of larger differences with decreasing gestational age at birth. At age 5 years, fewer children born SGA and moderate preterm had a normal BMI compared with those born AGA and late term.

Strengths and limitations. The main strength of the study was the large cohort size of 41,669 children. The cohort can be considered as population based, including all deliveries and child health centres in one county of Sweden. Antenatal, delivery and child health care is standardized and free of charge in Sweden, and inhabitants in Uppsala County have very high attendance to child health care ${ }^{18}$. The size of the cohort allowed us to divide the cohort into narrow strata of gestational age, and also use the $3^{\text {rd }}$ percentile as definition of SGA in the GLS model. Children born very preterm is a group with high co-morbidity. By excluding them, we could reduce the risk of exaggerating the potential impact of moderate to late preterm birth on postnatal growth and body proportions, often called type I error. Multiple pregnancy has a high level of co-morbidity and is one of the most common 


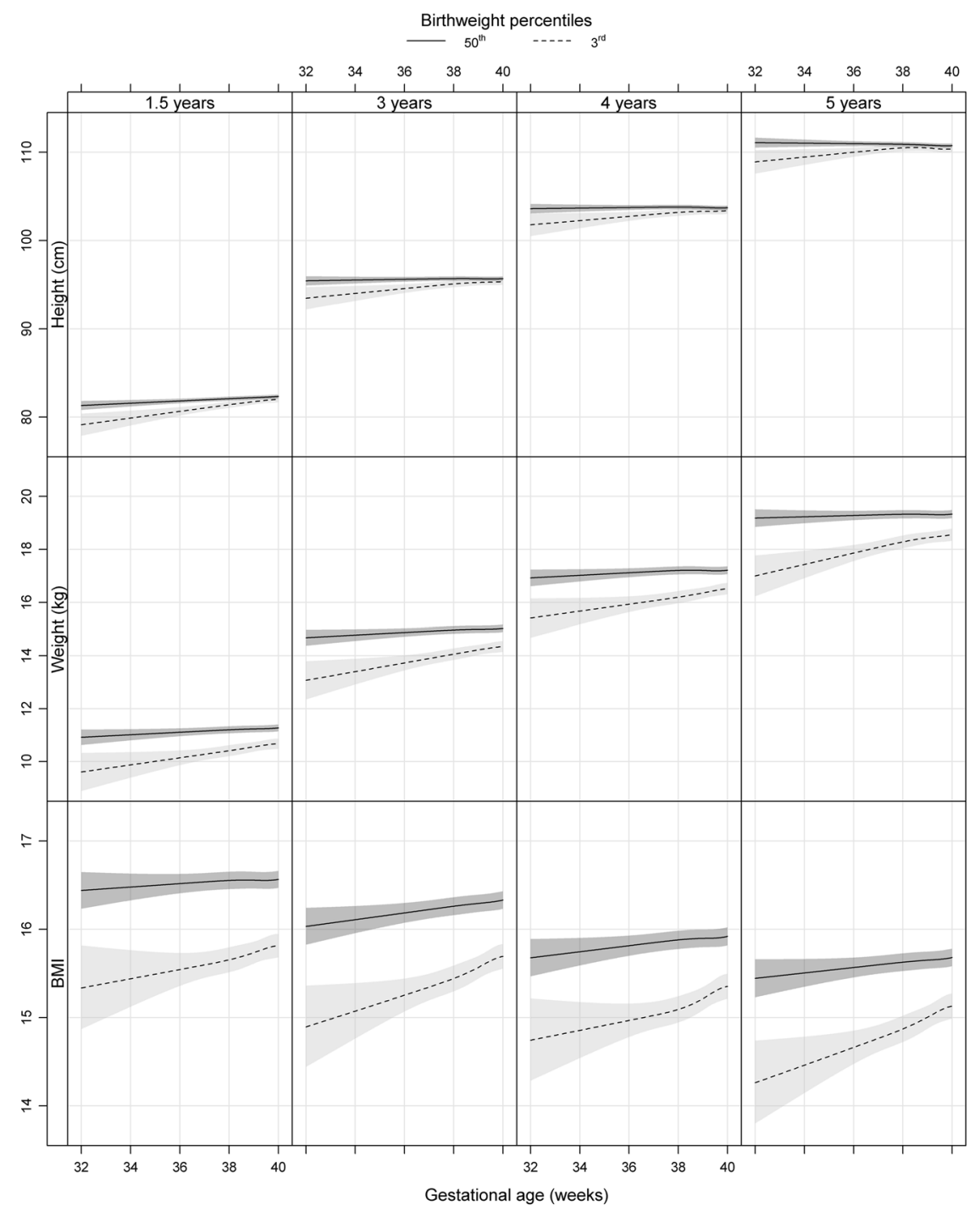

Figure 1. Estimated mean height, weight and BMI in children born standardized approptiate for gestational age (sAGA, birthweight $50^{\text {th }}$ percentile) and standardized small for gestational age (sSGA, birthweight $3^{\text {rd }}$ percentile) at age 1.5, 3, 4 and 5 years. Model adjusted for maternal height, BMI, diabetic disease, smoking habits at gestational week 32, country of birth, breastfeeding at age two months and gender. Significant differences are indicated by overlapping confidence intervals, which are presented in Tables 2 and 4.

etiological factors behind moderate to late preterm birth ${ }^{19}$. Therefore, we also excluded children born in multiple pregnancies. Anthropometric data was prospectively collected by a trained nurse, which minimizes the risk of recall and information bias. The linkage of data from several population based registries with information on socio-economic confounders as well as maternal and infant characteristics made it possible to adjust for several important confounders.

There are some important limitations of the study. First, despite the large cohort size, there were relatively few children born moderate preterm and SGA. Considering this, we could not calculate the proportion of children with a normal BMI at 5 years and use of the $3^{\text {rd }}$ percentile as cut-off. Second, data on some important potential confounders, most importantly breastfeeding, was incomplete. In order to use the whole dataset for the analyses, we imputed data on breastfeeding. Third, in order to guarantee the integrity of the study subjects, we did not have data on date of birth. Hence, we were not able to correct the age, at which anthropometric measurements were performed, for degree of prematurity. This might have an implication on the results, especially at age 1.5 years. However, any underestimation of childhood body size in children born preterm should be similar for those born SGA and AGA. Fourth, we lack data on paternal height, which influences growth in early childhood equally as maternal height ${ }^{20}$. Last, we did not have data on paternal BMI or eating habits of the families, which might be an important confounder for body proportion during childhood.

Comparison with earlier studies. In a Dutch study, they followed a cohort of comparable size to ours regarding growth in height and weight until four years of age ${ }^{21,22}$. In the first paper of the study, they showed that 


\begin{tabular}{|c|c|c|c|c|}
\hline & \multicolumn{2}{|l|}{ Height difference $^{\mathrm{a}}$} & \multicolumn{2}{|l|}{ Weight difference ${ }^{\mathrm{a}}$} \\
\hline & In cm $(95 \% \mathrm{CI})$ & In SD (95\% CI) & In $\mathrm{kg}(95 \% \mathrm{CI})$ & In SD $(95 \% \mathrm{CI})$ \\
\hline \multicolumn{5}{|l|}{1.5 years } \\
\hline \multicolumn{5}{|c|}{ Born $s$ SGA } \\
\hline 32 weeks & $-2.18(-3.49 ;-0.87)$ & $-0.78(-1.25 ;-0.31)$ & $-1.31(-2.06 ;-0.56)$ & $-1.09(-1.72 ;-0.47)$ \\
\hline 35 weeks & $-1.43(-2.07 ;-0.78)$ & $-0.51(-0.74 ;-0.28)$ & $-1.05(-1.42 ;-0.68)$ & $-0.88(-1.18 ;-0.57)$ \\
\hline 40 weeks & $-0.33(-0.60 ;-0.06)$ & $-0.12(-0.21 ;-0.02)$ & $-0.60(-0.75 ;-0.44)$ & $-0.50(-0.63 ;-0.37)$ \\
\hline \multicolumn{5}{|l|}{5 years } \\
\hline \multicolumn{5}{|c|}{ Born $s$ SGA } \\
\hline 32 weeks & $-2.18(-3.60 ;-0.75)$ & $-0.50(-0.82 ;-0.17)$ & $-2.18(-3.00 ;-1.36)$ & $-0.95(-1.30 ;-0.59)$ \\
\hline 35 weeks & $-1.27(-1.97 ;-0.57)$ & $-0.29(-0.45 ;-0.13)$ & $-1.61(-2.01 ;-1.20)$ & $-0.70(-0.87 ;-0.52)$ \\
\hline 40 weeks & $-0.37(-0.67 ;-0.07)$ & $-0.08(-0.15 ;-0.02)$ & $-0.78(-0.95 ;-0.60)$ & $-0.34(-0.41 ;-0.26)$ \\
\hline
\end{tabular}

Table 3. Differences in estimated mean height and weight. Children born standardized small for gestational age $(s$ SGA) in gestational weeks $32+0,35+0$ and $40+0$ compared with children born standardized appropriate for gestational age ( $s$ AGA) in the corresponding gestational week. $s$ AGA defined as birthweight $50^{\text {th }}$ percentile and sSGA as birthweight $3^{\text {rd }}$ percentile of expected for gestational age and sex according to the Swedish reference for birthweight ${ }^{35}$. ${ }^{\mathrm{a}}$ Model adjusted for maternal height, BMI, country of birth, maternal diabetic disease during pregnancy, smoking in pregnancy week 32 and breastfeeding at age two months.

\begin{tabular}{|l|l|l|}
\hline & \multicolumn{2}{|l|}{${\text { Adjusted } \text { BMI ratio }^{\mathbf{a}}}$} \\
\cline { 2 - 3 } & $\mathbf{1 . 5}$ years & $\mathbf{5}$ years \\
\hline sAGA 32/sAGA 40 & $0.99(0.98 ; 1.00)$ & $0.98(0.97 ; 1.00)$ \\
\hline sAGA 35/sAGA 40 & $1.00(0.99 ; 1.00)$ & $0.99(0.98 ; 1.00)$ \\
\hline sSGA 32/sAGA 40 & $0.93(0.90 ; 0.95)$ & $0.91(0.88 ; 0.94)$ \\
\hline sSGA 35/sAGA 40 & $0.94(0.92 ; 0.95)$ & $0.93(0.91 ; 0.94)$ \\
\hline sSGA 40/sAGA 40 & $0.95(0.95 ; 0.96)$ & $0.96(0.96 ; 0.97)$ \\
\hline sSGA 32/sAGA 32 & $0.93(0.90 ; 0.96)$ & $0.92(0.89 ; 0.96)$ \\
\hline sSGA 35/sAGA 35 & $0.94(0.92 ; 0.95)$ & $0.94(0.92 ; 0.95)$ \\
\hline
\end{tabular}

Table 4. Ratio of estimated mean BMI in children born standardized appropriate for gestational age ( $s$ AGA) or small for gestational age ( $s$ SGA) in week $32+0$ ( $s$ AGA 32 and sSGA 32 ), week $35+0$ (sAGA 35 and sSGA 35) or week $40+0$ ( $s$ AGA 40 and $s$ SGA 40$)$. sAGA defined as birthweight $50^{\text {th }}$ percentile and $s$ SGA as birthweight $3^{\text {rd }}$ percentile of expected for gestational age and sex according to the Swedish reference for birthweight ${ }^{35}$. ${ }^{\mathrm{a}}$ Model adjusted for maternal height, BMI, country of birth, maternal diabetic disease during pregnancy, smoking in pregnancy week 32 and breastfeeding at age two months.

without considering birthweight, children born in week 32-35 were shorter and lighter at 4 years than those born term. Moreover, among children born moderate preterm, those born SGA had an increased risk of short stature at 4 years compared with those born AGA. The second paper examined combined exposure to being born SGA or preterm (week 26-35). In conformity with our results, combined exposure had larger impact on growth in height and weight than isolated exposure. In the Dutch study, each study subject was measured on average 9.9 times. It is possible that largely deviant growth in a few subjects have large impact on the cohort mean as they did not account for repeated measurements. In our study we constructed a GLS model, where gestational age and standardized birthweight as well as postnatal anthropometrics were used as continuous variables. This allowed us to construct a prediction model with smoothed curves and more precise estimated means, which accounts for repeated measurements. Despite the different statistical methods, the results of the Dutch and our study point in the same direction and thus show reproducibility.

We found of a J-shaped pattern in BMI at 5 years in children born SGA and moderate preterm, with a tendency towards increased incidence of obesity. A large proportion had low BMI, which generates a low mean weight. This is in accordance with earlier studies of body proportions in adulthood in children born SGA or preterm, especially for children with short adult stature 23,24 . At 5 years of age, catch-up growth after being born SGA has usually already taken place ${ }^{3}$. Considering this, our finding of a shorter mean height of $2 \mathrm{~cm}$ at 5 years in children born SGA and moderate preterm might have important cognitive implications as more children will remain short. Even though evidence is limited for children born moderate preterm, the risk of adverse health outcome after rapid catch-up in weight or limited catch-up in height in early childhood indicates that postnatal growth patterns are of importance, and more research is needed in children born moderate preterm ${ }^{25,26}$.

In our study, we chose to use birthweight reference standards when we estimated birthweight for gestational age and sex. These references tend to underestimate the frequency of SGA in children born preterm ${ }^{27}$. Thereby, the difference between children born AGA and SGA might be underestimated. 


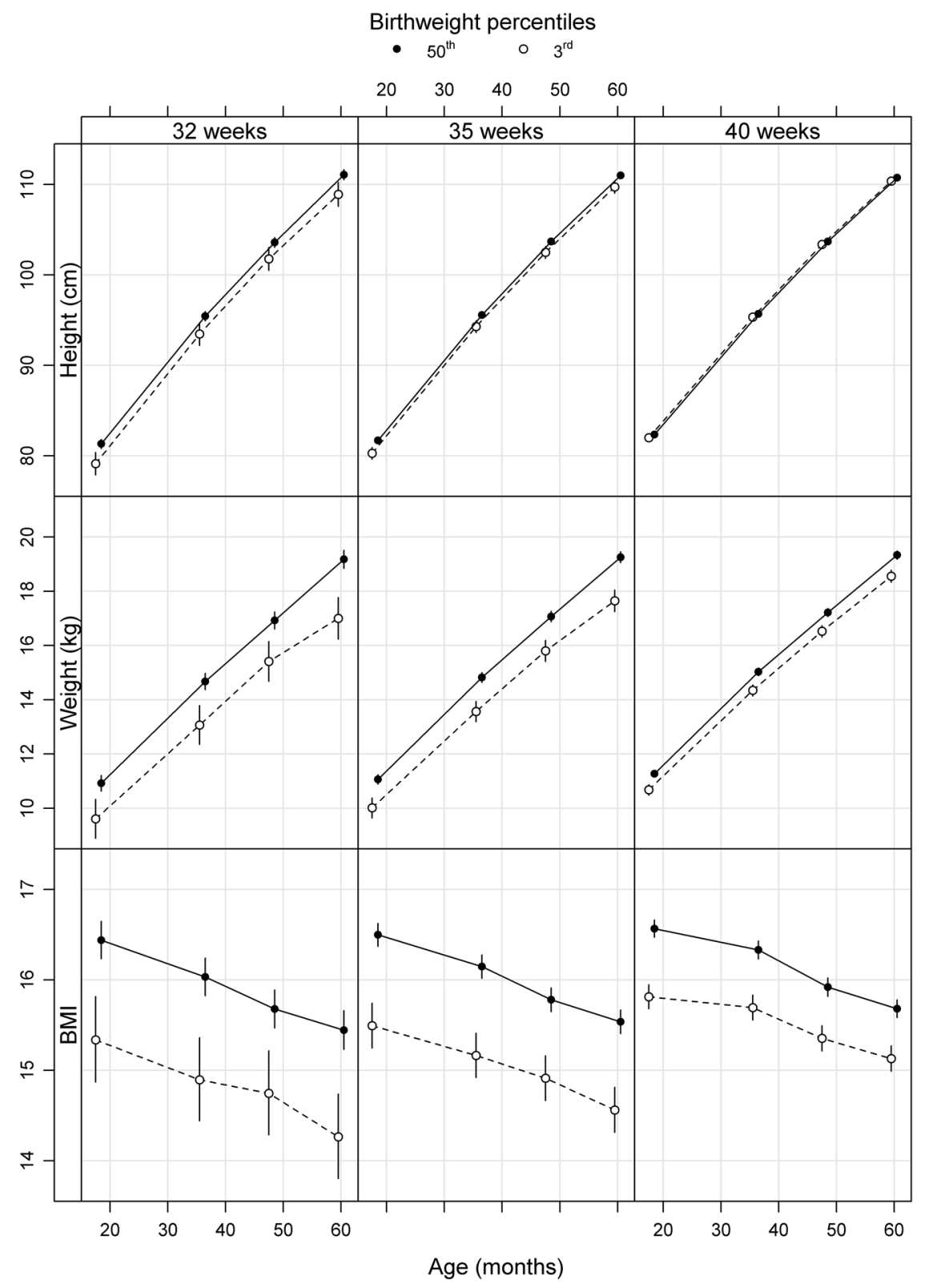

Figure 2. Estimated mean height, weight and BMI from age 1.5 to 5 years in children born standardized approptiate for gestational age (sAGA, birthweight $50^{\text {th }}$ percentile) and standardized small for gestational age (sSGA, birthweight $3^{\text {rd }}$ percentile) in gestational week $32+0,35+0$ and $40+0$. Model adjusted for maternal height, BMI, diabetic disease, smoking habits at gestational week 32, country of birth, breastfeeding at age two months and gender. Significant differences are indicated by overlapping confidence intervals, which are presented in Tables 3 and 4 .

Perspectives. After combined exposure to being born SGA and moderate to late preterm, body proportions differed from children with single exposure to SGA or preterm birth. As BMI is a measurement that includes height as well as weight, this shows that combined exposure not merely increases the risk of a slower growth, but also has an impact on body proportions. With height being largely genetically and growth hormone dependent, the growth hormone axis does not seem to be heavily influenced. However, we speculate that a J-shaped pattern of BMI at 5 years of age after being born SGA and moderate preterm might have an impact on metabolic and cardiovascular future health.

Andrews et al. showed that in children born very preterm, postnatal growth restriction can be less pronounced with improved early nutritional care ${ }^{28}$. Even though children born after week 32 suffer from less neonatal morbidity than those born very preterm, early feeding difficulties are still common. Fewer infants born preterm are breast-fed compared with those born term ${ }^{29,30}$. Although we do not known if feeding-practices in children born moderate preterm affect postnatal growth, we speculate that improved nutrition might limit neonatal growth restriction and, if born SGA, early catch-up growth might be enhanced. 


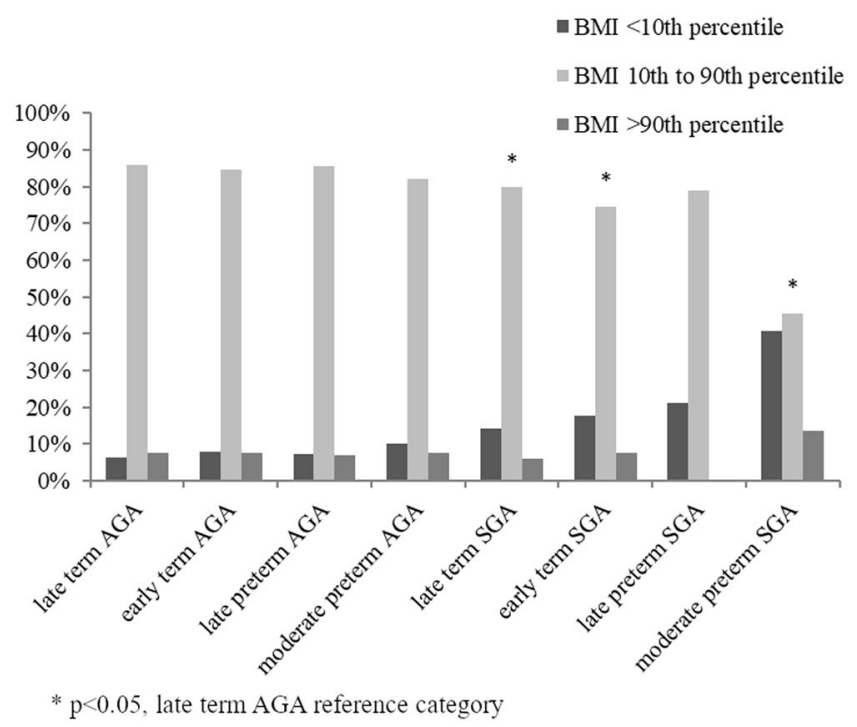

Figure 3. Proportion of children with low, normal and high BMI.

The potential metabolic risks but cognitive advantages of catch-up growth render difficulties in recommendations of appropriate nutrition during early infancy. There is a consensus on overall benefits of catch-up growth in preterm infants, which is mainly based on studies on infants born before 31 weeks ${ }^{4}$. However, despite slower weight-gain, very preterm born infants have neurocognitive benefits from breastfeeding ${ }^{31}$. In our study, being born moderate to late preterm and SGA, but not AGA, was associated with changes in body proportions. Unfortunately, we had too few subjects with complete data to explore if breastfeeding seemed to modify growth patterns. More research is needed to evaluate if postnatal growth patterns and long-term metabolic outcomes are affected by breastfeeding habits in children born moderate preterm ${ }^{32}$.

In conclusion, compared with being born AGA and term, being born SGA and moderate to late preterm is associated with shorter stature and lower BMI during the first five years of life. SGA status was found to have a greater impact on growth and body proportions than gestational age at birth. Thus, in the perspective of postnatal growth, children with a satisfactory intrauterine growth seem to cope with moderate to late preterm birth well. For children with insufficient intrauterine growth, there is a pattern of interaction with shorter stature and more abnormal body proportions the shorter gestational age.

\section{Methods}

Data sources. Uppsala County Mother and Child Database is a population based database which contains data from several national and regional registries. The included registries are the Medical Birth Register (MBR), Database of statistics of the Child Health Care Unit in Uppsala County, Register of Education and Register of Total Population. All children born at Uppsala University Hospital, the only hospital with a delivery unit in the county, who have later visited child health care in Uppsala County, Sweden, during the years 2000-2015 are included in the Uppsala County Mother and Child Database.

The MBR is a national registry and contains information on maternal characteristics, pregnancy complications, delivery, neonatal characteristics and complications of all pregnancies and births after 22 gestational weeks. It is based on mandatory registration of prospectively collected information. In Sweden, maternal, delivery and child health care are free of charge and follows a standardized schedule. The first antenatal visit usually takes place before 13 weeks of pregnancy, and involves an interview about medical and obstetric history, social situation including cohabitation and smoking habits three months before pregnancy and in early pregnancy ${ }^{33}$. The woman is weighed and her height is recorded. In pregnancy week 32, the woman is once again asked about her current smoking habits. Data is recorded using check-boxes, and anthropometric measurements are recorded in absolute numbers. Complications and diseases are classified using the International Classification of Diseases (ICD).

Data from child health care in Uppsala County is registered in the Database of statistics of the Child Health Care Unit in Uppsala County. 97\% of children living in Uppsala County regularly visit child health services during their first six years ${ }^{18}$. The registry contains information on anthropometric measurements and health data, as well as data from parental interviews about exclusive or partial breastfeeding at two months, and smoking habits at 4 months of age.

Data on maternal level of education is collected from the Register of Education, and maternal country of birth from the Register of Total Population. We used personal identification number assigned to all citizens in Sweden to cross-link data between the registries ${ }^{34}$.

Subjects. Uppsala County Mother and Child Database contains information on 57,044 children born in Uppsala County. All children had known sex. We excluded children with unknown gestational age $(\mathrm{n}=64)$ or birthweight $(\mathrm{n}=54)$. A birthweight of $<-5$ or $>5$ standard deviations (SD) of expected birth weight for gestational age and sex was considered misclassified and hence excluded $(n=27)$. Further we excluded children born 
after multiple pregnancies $(n=1629)$, with chromosome aberrations or malformations $(n=956)$, leaving 54,314 children in the study population. Since the aim was to study children with moderate to late preterm birth, we excluded children born with gestational age $<32$ weeks $(n=312)$ and $\geq 41$ weeks $(n=12,333)$. The final cohort consisted of 41,669 children.

Exposures. The studied exposures were gestational age and weight for gestational age and sex at birth. Gestational age was expressed as completed gestational weeks and days at birth. In the first model, gestational age was used as a continuous variable, and the outcome was estimated for children born with gestational age $32+0$, $35+0$ and $40+0$. Next, the cohort was stratified according to gestational age as moderate preterm (32-34 weeks), late preterm (35-36 weeks), early term (37-38 weeks) and late term (39-40 weeks). Most pregnancies, $>92 \%$, were dated with early second trimester ultrasound.

Birthweight for gestational age and sex was calculated using the national reference curve for birthweight ${ }^{35}$. We used two different definitions of being born SGA. First, with birthweight as a continuous exposure variable, where birthweight for gestational age and sex at the $3^{\text {rd }}$ percentile was defined as standardized SGA (sSGA). Second, we classified SGA as birthweight $<10^{\text {th }}$ percentile of expected.

Outcome. The main outcome was growth in height $(\mathrm{cm})$, weight $(\mathrm{kg})$ and BMI $\left(\mathrm{kg} / \mathrm{m}^{2}\right)$ during the first five years of childhood. Height and weight were measured at ages 1.5, 3, 4 and 5 years by a trained nurse in routine child health care. Since 2005, anthropometrics are registered electronically, thus data is missing for the younger ages in children born before 2003. Only measurements performed within 2 months from planned according to the age of the child are included in the Uppsala County Mother and Child Database.

Covariates. Maternal age, height, BMI, parity, parental cohabitation at first antenatal visit, maternal diabetic disease during pregnancy, smoking habits in pregnancy week 32, maternal country of birth, maternal level of education, male gender, Apgar score at $5 \mathrm{~min}$ and exclusive or partial breastfeeding at age two months were considered potential covariates. Maternal diabetic disease was identified by the corresponding ICD-10 codes (O241-O243, E10-14).

In order to select covariates for the multivariable models, we drew a directed acyclic graph (DAG). The minimal sufficient set for confounder adjustment included maternal height, BMI, diabetic disease, smoking habits at gestational week 32, country of birth and breastfeeding at age two months.

Statistical analysis. Clinical and demographic characteristics of the study population were cross-tabulated according to gestational age at birth, grouped as moderate preterm (32-34 weeks), late preterm (35-36 weeks), early term (37-38 weeks) and late term (39-40 weeks) birth. Children born late term were treated as reference group.

In order to account for the repeated measures in the outcome, we applied a generalized least squares model (GLS). Unlike generalized mixed effects model, GLS does not use random effects, but instead requires a specification of the within individual correlation structure. The regression model included the anthropometric measurements at each visit to child health care (age 1.5, 3, 4 and 5 years), but also the interaction between gestational age and standardized birthweight at birth. The model was adjusted for gender and potential confounders suggested by the DAG. All continuous variables were modelled using restricted cubic splines with 3-5 knots based on the apparent strength of association with the outcome. All models assumed a continuous autoregressive AR(1) correlation structure between the repeated measurements.

The GLS model described the growth trajectories according to standardized birthweight and gestational age. Using the GLS model, we estimated means of height, weight and BMI, both for children with birthweight $50^{\text {th }}$ percentile of expected for gestational age and sex (standardized AGA, sAGA) and $3^{\text {rd }}$ percentile (standardized SGA, $s S G A)$ at gestational week $32+0,35+0$ and $40+0$. We calculated the contrasts between estimated mean heights and mean weights with $95 \%$ confidence interval (CI), i.e. the difference between children born $s$ AGA or $s$ SGA at $32+0,35+0$ or $40+0$ gestational weeks and the reference value $(s$ AGA week $40+0)$. Next, we compared children born $s$ AGA with those born sSGA in the same gestational week. The contrasts were calculated as difference in $\mathrm{cm}$ or kg, but also in standard deviations according to the Swedish reference curve for height and weight ${ }^{36}$.

The contrast of estimated mean BMI was calculated as the ratio between the estimated mean BMI and the reference value of estimated mean BMI, e.g. a ratio of 0.95 indicates that the BMI was $5 \%$ lower than the reference value. First, we compared children born $s$ AGA or $s$ SGA in gestational week $32+0,35+0$ or $40+0$, with the reference $s$ AGA week $40+0$. In the next step, sSGA at $32+0,35+0$ and $40+0$ weeks were compared with $s$ AGA in the corresponding gestational week (reference value).

Incomplete baseline variables were imputed under fully conditional specification ${ }^{37}$. The outcome values were included in the imputation, but the imputed outcome values were subsequently discarded ${ }^{38}$. Model parameters were estimated in each imputed data set separately and their estimates and standard errors were combined using Rubin's rules. For comparison, a complete case analysis was also performed.

Lastly, we classified the cohort according to birthweight, as AGA or SGA, and gestational age (late term, early term, late preterm and moderate preterm). We used the anthropometric measurements at age 5 years to calculate the proportion of children with normal BMI and compared the groups using Chi-Square test.

IBM SPSS Statistics 2.5 and R version 3.5.0 using the mice and rms packages were used in the statistical calculations.

Research involving human participants. Ethic permission was approved by the Regional Ethical Review Board in Uppsala (no. 2012/410). All procedures involving human subjects were carried out in accordance with the ethical standards of the 1964 Helsinki declaration. All registry data was merged and de-identified by Statistics Sweden. Thereby, informed consent was not required. 


\section{Data Availability}

The datasets generated during and/or analyzed during the current study are not publicly available due to the ethical and legal restrictions prohibiting the sharing of personal data, but are available from the corresponding author on reasonable request.

\section{References}

1. Saenger, P., Czernichow, P., Hughes, I. \& Reiter, E. O. Small for gestational age: short stature and beyond. Endocrine reviews 28, 219-251, https://doi.org/10.1210/er.2006-0039 (2007).

2. de Bie, H. M. A., Oostrom, K. J. \& Delemarre-van de Waal, H. A. Brain Development, Intelligence and Cognitive Outcome in Children Born Small for Gestational Age. Hormone research in paediatrics 73, 6-14 (2010).

3. Karlberg, J. \& Albertsson-Wikland, K. Growth in full-term small-for-gestational-age infants: from birth to final height. Pediatr Res 38, 733-739, https://doi.org/10.1203/00006450-199511000-00017 (1995).

4. Singhal, A. Long-Term Adverse Effects of Early Growth Acceleration or Catch-Up Growth. Annals of nutrition \& metabolism 70, 236-240, https://doi.org/10.1159/000464302 (2017).

5. Lundgren, E. M., Cnattingius, S., Jonsson, B. \& Tuvemo, T. Intellectual and psychological performance in males born small for gestational age with and without catch-up growth. Pediatr Res 50, 91-96, https://doi.org/10.1203/00006450-200107000-00017 (2001).

6. Martin, A., Connelly, A., Bland, R. M. \& Reilly, J. J. Health impact of catch-up growth in low-birth weight infants: systematic review, evidence appraisal, and meta-analysis. Maternal \& child nutrition 13, https://doi.org/10.1111/mcn.12297 (2017).

7. Nam, H. K. \& Lee, K. H. Small for gestational age and obesity: epidemiology and general risks. Annals of pediatric endocrinology \& metabolism 23, 9-13, https://doi.org/10.6065/apem.2018.23.1.9(2018).

8. Ray, J. G., Park, A. L. \& Fell, D. B. Mortality in Infants Affected by Preterm Birth and Severe Small-for-Gestational Age Birth Weight. Pediatrics 140, https://doi.org/10.1542/peds.2017-1881 (2017).

9. Nobile, S., Marchionni, P. \& Carnielli, V. P. Neonatal outcome of small for gestational age preterm infants. European journal of pediatrics 176, 1083-1088, https://doi.org/10.1007/s00431-017-2957-1 (2017).

10. Shapiro-Mendoza, C. K. \& Lackritz, E. M. Epidemiology of late and moderate preterm birth. Seminars in fetal \& neonatal medicine 17, 120-125, https://doi.org/10.1016/j.siny.2012.01.007 (2012).

11. Cooke, R. J., Ainsworth, S. B. \& Fenton, A. C. Postnatal growth retardation: a universal problem in preterm infants. Archives of disease in childhood. Fetal and neonatal edition 89, F428-430, https://doi.org/10.1136/adc.2001.004044 (2004).

12. Cole, T. J., Statnikov, Y., Santhakumaran, S., Pan, H. \& Modi, N. Birth weight and longitudinal growth in infants born below 32 weeks' gestation: a UK population study. Archives of disease in childhood. Fetal and neonatal edition 99, F34-40, https://doi. org/10.1136/archdischild-2012-303536 (2014).

13. Ong, K. K. et al. Postnatal growth in preterm infants and later health outcomes: a systematic review. Acta paediatrica (Oslo, Norway: 1992) 104, 974-986, https://doi.org/10.1111/apa.13128 (2015).

14. Taine, M. et al. Early postnatal growth and neurodevelopment in children born moderately preterm or small for gestational age at term: A systematic review. Paediatric and perinatal epidemiology 0, https://doi.org/10.1111/ppe.12468.

15. Itabashi, K. et al. Longitudinal follow-up of height up to five years of age in infants born preterm small for gestational age; comparison to full-term small for gestational age infants. Early human development 83, 327-333, https://doi.org/10.1016/j. earlhumdev.2006.07.002 (2007).

16. Sullivan, M. C., McGrath, M. M., Hawes, K. \& Lester, B. M. Growth trajectories of preterm infants: birth to 12 years. Journal of pediatric health care: official publication of National Association of Pediatric Nurse Associates \& Practitioners 22, 83-93, https://doi. org/10.1016/j.pedhc.2007.02.008 (2008).

17. Fellman, V. et al. Incidence of and risk factors for neonatal morbidity after active perinatal care: extremely preterm infants study in Sweden (EXPRESS). Acta paediatrica (Oslo, Norway: 1992) 99, 978-992, https://doi.org/10.1111/j.1651-2227.2010.01846.x (2010).

18. Wallby, T. \& Hjern, A. Child health care uptake among low-income and immigrant families in a Swedish county. Acta paediatrica (Oslo, Norway: 1992) 100, 1495-1503, https://doi.org/10.1111/j.1651-2227.2011.02344.x (2011).

19. Refuerzo, J. S. Impact of multiple births on late and moderate prematurity. Seminars in fetal \& neonatal medicine 17, 143-145, https://doi.org/10.1016/j.siny.2012.01.012 (2012).

20. Griffiths, L. J., Dezateux, C. \& Cole, T. J. Differential parental weight and height contributions to offspring birthweight and weight gain in infancy. International journal of epidemiology 36, 104-107, https://doi.org/10.1093/ije/dyl210 (2007).

21. Bocca-Tjeertes, I. F., Kerstjens, J. M., Reijneveld, S. A., de Winter, A. F. \& Bos, A. F. Growth and predictors of growth restraint in moderately preterm children aged 0 to 4 years. Pediatrics 128, e1187-1194, https://doi.org/10.1542/peds.2010-3781 (2011).

22. Bocca-Tjeertes, I. F., Reijneveld, S. A., Kerstjens, J. M., de Winter, A. F. \& Bos, A. F. Growth in small-for-gestational-age pretermborn children from 0 to 4 years: the role of both prematurity and SGA status. Neonatology 103, 293-299, https://doi. org/10.1159/000347094 (2013).

23. Lundgren, E. M., Cnattingius, S., Jonsson, B. \& Tuvemo, T. Prediction of adult height and risk of overweight in females born smallfor-gestational-age. Paediatric and perinatal epidemiology 17, 156-163 (2003).

24. Derraik, J. G., Lundgren, M., Cutfield, W. S. \& Ahlsson, F. Association Between Preterm Birth and Lower Adult Height in Women. American journal of epidemiology 185, 48-53, https://doi.org/10.1093/aje/kww116 (2017).

25. Lundgren, E. M., Cnattingius, H. M., Jonsson, G. B. \& Tuvemo, T. H. Linear catch-up growth does not increase the risk of elevated blood pressure and reduces the risk of overweight in males. Journal of hypertension 19, 1533-1538 (2001).

26. Kerkhof, G. F., Willemsen, R. H., Leunissen, R. W., Breukhoven, P. E. \& Hokken-Koelega, A. C. Health profile of young adults born preterm: negative effects of rapid weight gain in early life. The Journal of clinical endocrinology and metabolism 97, 4498-4506, https://doi.org/10.1210/jc.2012-1716 (2012).

27. Cooke, R. W. Conventional birth weight standards obscure fetal growth restriction in preterm infants. Archives of disease in childhood. Fetal and neonatal edition 92, F189-192, https://doi.org/10.1136/adc.2005.089698 (2007).

28. Andrews, E. T., Ashton, J. J., Pearson, F., Beattie, R. M. \& Johnson, M. J. Early postnatal growth failure in preterm infants is not inevitable. Archives of Disease in Childhood - Fetal and Neonatal Edition, https://doi.org/10.1136/archdischild-2018-315082 (2018).

29. Williams, J. E. \& Pugh, Y. The Late Preterm: A Population at Risk. Critical care nursing clinics of North America 30, 431-443, https:// doi.org/10.1016/j.cnc.2018.07.001 (2018).

30. Ayton, J., Hansen, E., Quinn, S. \& Nelson, M. Factors associated with initiation and exclusive breastfeeding at hospital discharge: late preterm compared to 37 week gestation mother and infant cohort. International breastfeeding journal 7, 16, https://doi. org/10.1186/1746-4358-7-16 (2012).

31. Roze, J. C. et al. The apparent breastfeeding paradox in very preterm infants: relationship between breast feeding, early weight gain and neurodevelopment based on results from two cohorts, EPIPAGE and LIFT. BMJ open 2, e000834, https://doi.org/10.1136/ bmjopen-2012-000834 (2012).

32. Boquien, C. Y. Human Milk: An Ideal Food for Nutrition of Preterm Newborn. Frontiers in pediatrics 6, 295, https://doi.org/10.3389/ fped.2018.00295 (2018). 
33. Welfare, N. B. o. H. a. The Swedish Medical Birth Register-A Summary of Content and Quality. Report No. 2003-112-3, (National Board of Health and Welfare, Stockholm, Sweden, 2003).

34. Ludvigsson, J. F., Otterblad-Olausson, P., Pettersson, B. U. \& Ekbom, A. The Swedish personal identity number: possibilities and pitfalls in healthcare and medical research. European journal of epidemiology 24, 659-667, https://doi.org/10.1007/s10654-0099350-y (2009).

35. Niklasson, A. et al. An update of the Swedish reference standards for weight, length and head circumference at birth for given gestational age (1977-1981). Acta paediatrica Scandinavica 80, 756-762 (1991).

36. Wikland, K. A., Luo, Z. C., Niklasson, A. \& Karlberg, J. Swedish population-based longitudinal reference values from birth to 18 years of age for height, weight and head circumference. Acta paediatrica (Oslo, Norway: 1992) 91, 739-754 (2002).

37. van Buuren, S., Brand, J. P. L., Groothuis-Oudshoorn, C. G. M. \& Rubin, D. B. Fully conditional specification in multivariate imputation. J. Stat. Comput. Simul. 76, 1049-1064 (2006).

38. Hippel, P. Regression with Missing Y's: An Improved Strategy for Analyzing Multiply Imputed Data. Sociaological Methodology 37(1) (2007).

\section{Acknowledgements}

L.L. is supported by the Gillbergska foundation and Födelsefonden. A.K.W. is supported by the Swedish Research Council (Grant 2014-3561). F.A. is supported by the Gillbergska foundation and HRH Crown Princess Lovisa's association for paediatric care. All authors have read and approved the submission of the manuscript; the manuscript is original, has not been published and is not being considered for publication elsewhere, in whole or in part, in any language, except as an abstract. Open access funding provided by Uppsala University.

\section{Author Contributions}

Study concept and design: L.L., F.A., M.L., E.B., E.L. and A.-K.W. Statistical analyses: L.L. and E.L. Analysis and interpretation of data: L.L., F.A., E.L. and A.-K.W. First draft and revision of the manuscript: L.L. Critical revision of the manuscript for important intellectual content: L.L., F.A., M.L., E.B., E.L. and A.-K.W.

\section{Additional Information}

Competing Interests: The authors declare no competing interests.

Publisher's note: Springer Nature remains neutral with regard to jurisdictional claims in published maps and institutional affiliations.

Open Access This article is licensed under a Creative Commons Attribution 4.0 International License, which permits use, sharing, adaptation, distribution and reproduction in any medium or format, as long as you give appropriate credit to the original author(s) and the source, provide a link to the Creative Commons license, and indicate if changes were made. The images or other third party material in this article are included in the article's Creative Commons license, unless indicated otherwise in a credit line to the material. If material is not included in the article's Creative Commons license and your intended use is not permitted by statutory regulation or exceeds the permitted use, you will need to obtain permission directly from the copyright holder. To view a copy of this license, visit http://creativecommons.org/licenses/by/4.0/.

(C) The Author(s) 2019 